\title{
Effects of Sleep and Wakefulness upon Antidromic Responsiveness of the Lateral Geniculate Body
}

\author{
Kitsuya Iwama and Takuji Kasamatsu \\ Laboratory of Neurophysiology, Institute of Higher Nervous Activity, \\ Osaka University Medical School, Osaka
}

\begin{abstract}
Changes in the antidromic responsiveness of the cells of the lateral geniculate body (LGB) to single or repetitive stimulation of the optic radiation were studied in free behaving cats during the natural sleep-wakefulness cycle. The antidromic cellular response was minimum during light sleep (high voltage slow wave sleep) and increased during deep sleep (low voltage fast wave sleep). The responsiveness during resting arousal was intermediate between those during light and deep sleep. The deep sleep wave, a peculiar spontaneous electrical activity seen in the LGB during deep sleep, was found to intensify responsiveness of the LGB cells to antidromic stimulation.
\end{abstract}

The problem as to how responsiveness of the cells of the lateral geniculate body (LGB) changes during the natural sleep-wakefulness cycle has been attacked by a number of workers. ${ }^{3,4,7,11}$ Evidence, both direct and indirect, has accumulated indicating that the orthodromic responsiveness of the LGB cells to stimulation of the optic nerve or tract is minimum during light sleep (highvoltage slow wave sleep) and increases when the cortical EEG is showing a desynchronized pattern associated with either resting arousal or deep sleep (lowvoltage fast wave sleep). Among the two behavioral stages equally characterized with the desynchronized cortical EEG, deep sleep provides the LGB cells with stronger responsiveness than does resting arousal.

In the present study an attempt was made to reveal changes in responsiveness of the LGB cells, associated with the sleep-wakefulness transition, by stimulating the optic radiation antidromically. The results obtained have been found complementary to those obtained previously by the method of orthodromic stimulation.

\section{METHODS}

Cats provided with implanted electrodes in the sensorimotor cortex, the LGB and the optic chiasm were used. As to the type of the electrode at each site and the method of implanting it, readers are referred to our previous papers. ${ }^{4,6,8}$ In addition to these electrodes the cats had another pair of implanted electrodes

Received for publication, August 13, 1966. 
in the optic radiation. These radiation electrodes were two insulated wires, aligned singly with bare tips $1 \mathrm{~mm}$ apart. The electrode tips were positioned at the site with the Horsely-Clarke coordinates of $L 8$ and $A$ 4.5. The dorsoventral depth was unidentified, but it was adjusted so as to obtain the response of the LGB to single shock stimulation of the optic radiation with the pattern as will be described below.

In all the experiments the cats were placed in an observation box and allowed to behave free. The experimental room was kept dark and quiet.

\section{Results}

\section{1) Potential pattern of the antidromic $L G B$ response}

In the present study it was always ascertained that the electrodes of the LGB could display the response to single shock stimulation of the optic chiasm with the potential pattern which has been accepted as standard by a number of workers.1,9,10 As is shown in Fig. 1- $A$, right, the orthodromic response consists of a positive-negative or sometimes positive-negative-positive spike and a following negative spike of a relatively long duration (the polarity of the LGB response is
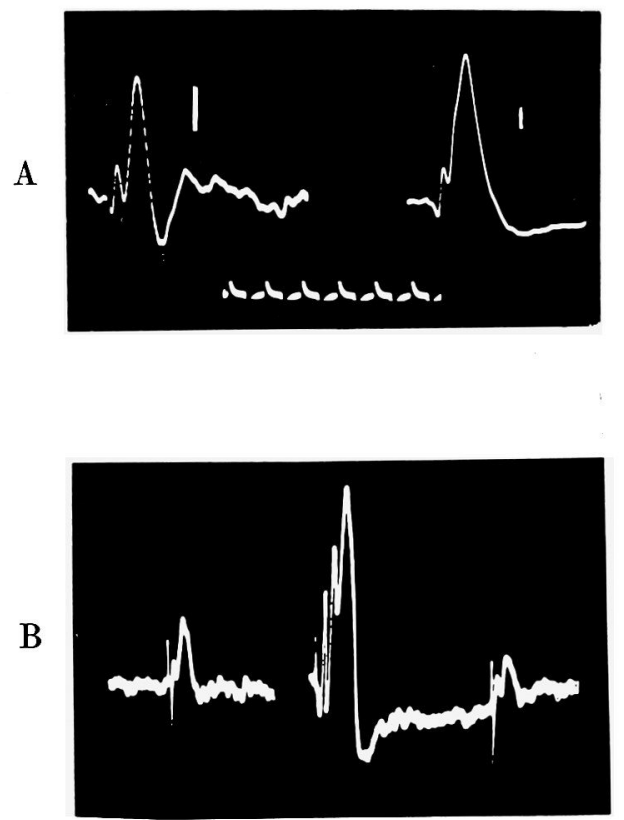

Fig. 1. Anti-and orthodromic responses of the LGB and their interaction. $A$, antidromic response (left) and orthodromic one (right). Voltage and time scales, $100 \mu \mathrm{V}$ and 1 msec. $B$, control antidromic response (left) and interaction of ortho- and antidromic stimulation at an interval of $6.7 \mathrm{msec}$ (right).

In this and all subsequent figures, electronegativity of the deeper tip of the bipolar LGB electrode is indicated upward. 
expressed in terms of electro-positivity or -negativity at the deeper tip of singly aligned bipolar electrodes). They have been interpreted as representing the action potential of the optic tract fiber terminals and the synchronized discharge of the postsynaptic LGB cells, respectively.

In the same animal and through the same LGB electrodes the response to single shock stimulation of the optic radiation appeared with the potential pattern as shown in the record of Fig. 1- $A$, left. The response was composed of two successive negative spikes, one having its peak at $0.3-0.4 \mathrm{msec}$ and the other at $0.8-0.9$ msec. after a stimulus artefact. In good recordings where the shock artefact was minimized, it was seen that the first negative spike occurred with a latency of about 0.1 msec. Though the relative magnitude of the two negative spikes was variable from case to case, it was usual that the first spike was smaller than the second one.

Our tentative interpretation of the potential pattern of the antidromic LGB response is as follows: the first negative spike is most probably a sign of antidromically conducted axonal spikes, whereas the second one is due to a synchronized discharge of the cell bodies activated antidromically. That the second negative spike is ascribable to the cellular discharge may be supported by the following findings: 1) When an antidromic response was preceded by an orthodromic one at short time intervals, this component was more or less suppressed. This is seen in the records of Fig. 1- $B$ where the ortho- and antidromic stimuli were applied at an interval of $6.7 \mathrm{msec}$. It is notable in these records that in the antidromic response conditioned by the orthodromic stimulation the second component was reduced markedly, whereas the first one was more or less augmented. This suggests that the two components of the antidromic response are different from each other in origin. A similar finding has been reported by Vastola ${ }^{10}$ who made a systematic study on interaction between ortho- and antidromic stimulation at the LGB. 2) Attempts were made in two acute preparations to pick up unitary discharges with microelectrodes. By applying a single shock to approximately the same locus as in the chronic preparation, evoked unitary spikes were searched for in the LGB and their latencies were measured. A total of 11 units were recorded, and it was found that the average latency was $0.91 \pm 0.43$ (S.D.) msec. Since this average latency determined at the unitary level is very close to the peak time of the second negative spike of the mass response, it may be justified to consider that this negative component is an aggregate of cellular discharges.

There is no direct evidence that the first component of the antidromic mass response is of axonal origin. However, since this component precedes the cellular discharge, it is most probable that the former signals the event leading to the latter. We, therefore, assume that the discharge of the most proximal part of the axon produces the electronegativity at the recording electrode as does 
the cellular discharge.

Vastola ${ }^{10}$ published records of the antidromic LGB response obtained from various depths in and around the LGB. The potential pattern in our recording is similar to that obtained by Vastola from the dorsal margin of the LGB (Fig. 1, A13 and Fig. 5 in his paper). The peak-to-peak interval between the two negative spikes, which we presume to represent the invasion time of antidromic impulses form the most proximal part of the axon to the cell body, is measured as $\mathbf{0 . 4 - 0 . 5}$ msec in Vastola's records. This value is about the same as found in our recording.

\section{2) Changes of the antidromic LGB response in sleep and wakefulness}

To follow changes in the antidromic responsiveness of the LGB cells during the sleep-wakefulness cycle, we divided the behavioral state into three, i.e., resting arousal, light sleep and deep one, on the basis of electrographic signs of the sensorimotor cortex and the posterior neck muscles. In each specified state of behavior a number of the LGB responses were recorded by applying to the optic radiation a single shock of a fixed intensity. Routinely, this procedure was followed by recording the same number of the LGB responses to single shock stimulation of the optic chiasm. In both cases of stimulation the applied electrical shock was made as brief as 0.01 msec and the stimulus intensity was kept submaximal.

An example of the records is shown in Fig. 2. All the records were made by superimposing 10 sweeps. First, it is seen in the top row of the figure that the

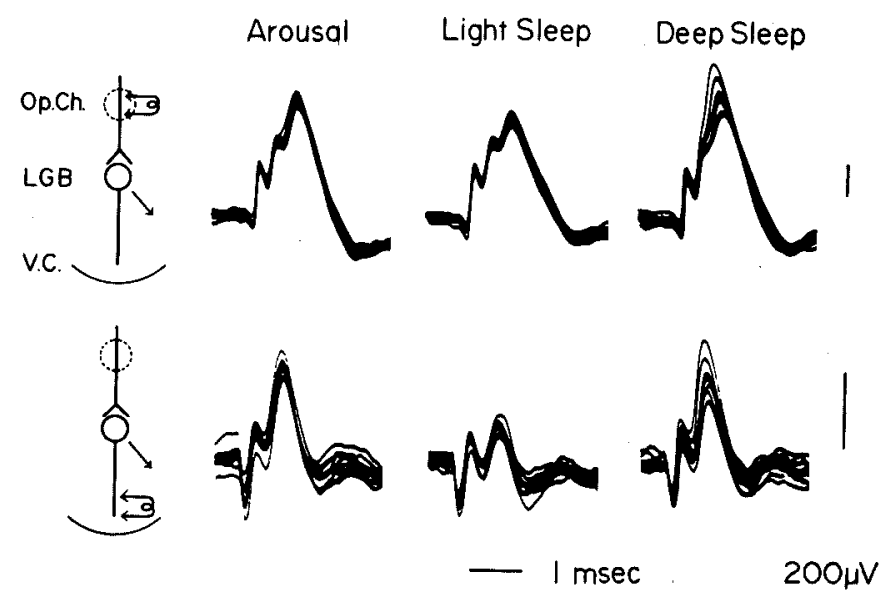

Fig. 2. Ortho- and antidromic responses of the LGB during resting arousal, light sleep and deep one. Top row, orthodromic responses. Bottom row, antidromic responses. The very early part of the first negative deflection of antidromic response was distorted by shock artefacts.

To the left, arrangement of electrodes for stimulation and recording is schematically shown. Op. Ch., optic chiasm. V.C., visual cortex. 
postsynaptic discharge to the orthodromic stimulation decreased when the cat entered light sleep from resting arousal, and upon precipitation of deep sleep it increased enormously above the level maintained during resting arousal. This finding is in complete agreement with our previous observation ${ }^{4,6}$ and with the inference of the previous workers based upon the indirect evidence. ${ }^{3,7,11}$

The bottom row of Fig. 2 is to show changes in the antidromic response recorded in the same behavioral state as the orthodromic response was examined. One can see that the second component of the response, which represents the discharge of the LGB cells, was minimum during light sleep, and increased exceeding the level maintained during resting arousal after deep sleep had been established. There were found no marked changes in the axonal discharge. But it is notable that the peak-to-peak interval between the axonal and cellular activities descreased as the cellular discharge increased in amplitude. This suggests that a decrease in the invasion time of antidromic impulses is caused by the same mechanism acting to increase the amplitude of the cellular discharge.

It is now evident that the antidromic stimulation reveals changes in responsiveness of the LGB cells associated with the sleep-wakefulness transition in the same way as does the orthodromic stimulation. However, it is our impression that differences in the cellular responsiveness between the three behavioral states appear more markedly in the antidromic stimulation than in the orthodromic stimulation. For example, the records of Fig. 2 shows that the relative increase of the cellular responsiveness in transition from light sleep to deep one is about $60 \%$ in the orthodromic stimulation, whereas the corresponding figure in the antidromic stimulation reaches as high as $130 \%$. This point will be discussed later.

\section{3) Facilitation of the antidromic $L G B$ response by the deep sleep wave}

It has been well-established that when cats are in the state of deep sleep there appear spontaneously slow waves of variable amplitudes in the LGB, the pontine reticular formation and some other subcortical structures. ${ }^{2,5}$ This slow wave, called deep sleep wave by Brooks and Bizzi, ${ }^{5}$ is found to associate with phasic movements of body and eyes, which are one of the characteristic signs of deep sleep. Previously, Iwama and his associates ${ }^{4,6,8}$ reported that in the presence of the deep sleep wave the orthodromic responsiveness of the LGB cells and the presynaptic inhibition at the optic tract fiber terminals were more or less intensified as compared with the levels in the phase of deep sleep lacking this peculiar spontaneous activity.

The experiment of Fig. 3 was conducted to see how the deep sleep wave modifies the antidromic response of the LGB cells. In this experiment the optic radiation was stimulated continuously at 100 cycles per sec with a submaximal intensity. It was ascertained that this repetitive stimulation of the optic radiation 

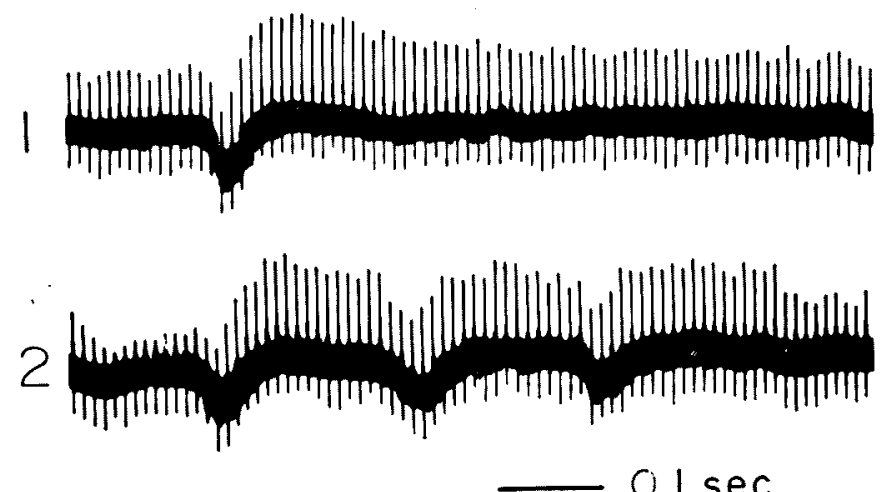

Fig. 3 Facilitation of antidromic LGB response by deep sleep wave. LGB was stimulated at 100 cycles per sec. Cellular component of antidromic response was shown as sharp upward spikes. Deep sleep wave was signaled by a downward excursion of the baseline followed by an upward deflection of low amplitude.

had no serious disturbances for maintaining deep sleep and that the LGB cells exhibited no marked signs of decreased responsiveness in a continuation of highfrequency stimulation.

It is seen from record 1 of Fig. 3 that when a singly isolated deep sleep wave appeared in the LGB as indicated by a downward positive deflection of the baseline, the antidromic response of the LGB cells began to increase in amplitude at the peak of the deep sleep wave and continued to be enhanced for 300 msec thereafter. The maximal enhancement was seen at the peak of the negative slow deflection. When the deep sleep wave occurred repetitively in bursts, it was seen that the antidromic LGB response was repeatedly enhanced in response to each deep sleep wave (record 2 of Fig. 3).

With the orthodromic LGB response a similar observation on the modulatory effect of the deep sleep wave was done by stimulating the optic chiasm at a high frequency. Confirming our previous observation, it was found that the orthodromically elicited discharge of the LGB cells was facilitated when it collided the deep sleep wave. It was noted in this observation that the time course of the facilitation was much the same as seen in the antidromic response. However, it was also noted that the facilitation of the testing response by the deep sleep wave appeared less marked in the orthodromic stimulation than in the antidromic one.

\section{Discussion}

Assuming that the component of the cellular activity in the antidromic LGB response is simply an aggregate of evoked unitary discharges, one can say that an increase or decrease in the amplitude of this component may signify an increase 
or decrease in the number of cells which are fully invaded by antidromic impulses. Whether or not antidromic impulses can invade individual cell bodies may primarily depend upon the degree of membrane depolarization in each cell. It is, therefore, likely that changes in the antidromic responsiveness of the LGB cells, associated with the natural sleep-wakefulness cycle, are a reflection of changes in membrane depolarization of the cell bodies of the LGB neurons. It is thus said that membrane depolarization of the LGB cells is strongest during deep sleep and decreases in the order from deep sleep to resting arousal and further to light sleep. Following this argument, one can say that the facilitation of the antidromic response by the deep sleep wave is ascribable to a depolarizing action of the latter exerted upon the cell bodies of the LGB.

Membrane depolarization of the cell bodies is also a factor determining whether or not the cell bodies are discharged transsynaptically. Therefore, it is very conceivable that in the sleep-wakefulness transition as well as in the presence of the deep sleep wave, responsiveness of the LGB cells to the orthodromic stimulation has been found to change exactly in parallel with that to the antidromic stimulation.

It was noted that an increase of the orthodromic responsiveness in shifting from light sleep to deep one or to resting arousal appears less marked than that seen in the antidromic responsiveness in the same transition of sleep and wakefulness. This is probably because the presynaptic inhibition of the optic tract fiber terminals is strengthened during deep sleep or resting arousal above the level during light sleep; though responsiveness of the postsynaptic cells is increased in shifting from light sleep, there is a concomitant increase in the presynaptic inhibition, so that orthodromic impusles are decreased at the presynaptic terminals and the final effect to discharge the postsynaptic cells becomes relatively less manifest. This same explanation will fit the finding that the facilitatory effect of the deep sleep wave upon responsiveness of the LGB cells is relatively less marked in testing by the orthodromic stimulation than by antidromic one, because there has been presented evidence that an increase in the postsynaptic responsiveness due to the deep sleep wave is associated with a concomitant increase in the presynaptic inhibition at the optic tract fiber terminals. ${ }^{4,6,8}$

In examining the facilitatory effect of the deep sleep wave upon responsiveness of the LGB cells, it was found that a facilitation of the testing response started at the peak of the positive deflection of the deep sleep wave. Previously, Sakakura and Iwama $^{8}$ made a similar observation on the effect of the deep sleep wave upon the presynaptic inhibition of the LGB, taking the antidromically elicited spike potential of the optic tract as an index. They found that the antidromic spike potential of the optic tract began to augment exactly in time with the start of the positive deflection of the deep sleep wave. Therefore, there is a suggestion that the action of the deep sleep wave upon the presynaptic 
terminals is different from that upon the postsynaptic cells at least in time course.

\section{Acknowledgment}

Our sincere thanks are due to Dr. Yasumasa Hayashi who kindly read the manuscript.

\section{References}

1) Bishop, P.O. \& Macleod, J.G. Nature of potential associated with synaptio transmission in lateral geniculate body of cat. J. Neurophysiol., 1954, 17, 387-414.

2) Brooks, D.C. \& Bizzi, E. Brainstem electrical activity during deep sleep. Arch. ital. Biol., 1963, 101, 648-665.

3) Dagnino, N., Favale, E., Loeb, C. \& Manfredi, N. Sensory transmission in the geniculostriate system of the cat during natural sleep and arousal. J. Neurophysiol., $1965,28,443-456$.

4) Iwama, K., Kawamoto, T., Sakakura, H. \& Kasamatsu, T. Responsiveness of cat lateral geniculate at pre- and postsynaptic levels during natural sleep. Physiol. \& Behav., 1966, 1, 45-53.

5) Jouvet, M. Telencephalic and rhombencephalic sleep in the cat. In: The Nature of Sleep, edited by G.E.W. Wolstenholme \& M. O'Connor, J. \& A. Churchill Ltd., London, 1961, p. 188.

6) Kasamatsu, T. \& Iwama, K. Two types of light sleep and central visual function in cats. Tohoku J. exp. Med., 1966, 88, 289-303.

7) Palestini, M., Pisano, M., Rosadini, G. \& Rossi, G.F. Visual cortical responses evoked by stimulating lateral geniculate body and optic radiation in awake and sleeping cats. Exp. Neurol., 1964, 8, 17-30.

8) Sakakura, H. \& Iwama, K. Presynaptic inhibition and postsynaptic facilitation in lateral geniculate body and so-called deep sleep wave activity. Tohoku J. exp. Med., $1965,87,40-51$.

9) Suzuki, H. \& Taira, N. Effect of reticular stimulation upon synaptic transmission in eat's lateral geniculate body. Jap. J. Physiol., 1961, 11, 641-655.

10) Vastola, E.F. Antidromic action potential in lateral geniculate body. $J$. Neurophysiol., 1957, 20, 167-185.

11) Walsh, J.J. \& Cordeau, J.P. Responsiveness in the visual system during various phases of sleep and waking. Exp. Neurol., 1965, 11, 80-103. 